\title{
Tree-Based Sequential Sampling Algorithm for Scalable Macromodeling of High-Speed Systems
}

\author{
Krishnan Chemmangat, Francesco Ferranti, Tom Dhaene and Luc Knockaert \\ Department of Information Technology, Ghent University - IBBT, \\ Gaston Crommenlaan 8, Bus 201, B-9050, Gent, Belgium. \\ email: $\{$ krishnan.cmc, francesco.ferranti, luc.knockaert, tom.dhaene $\} @$ intec.ugent.be
}

\begin{abstract}
This paper describes a new sequential sampling algorithm for efficient and accurate scalable macromodeling of parameterized frequency responses of high-speed systems. The proposed algorithm divides the design space into hyper-rectangular cells. A pertinent numerical example validates the capability of the proposed sequential sampling method.
\end{abstract}

\section{Introduction}

The design of high-speed microwave systems often requires multiple electromagnetic (EM) simulations to perform different design activities such as design space exploration, sensitivity analysis and design optimization, which is computationally very expensive. An alternative approach is to generate accurate scalable macromodels, which capture the parameterized frequency behavior of these systems with respect to design parameters, such as layout and substrate features. These scalable macromodels are computationally very cheap compared to EM simulators, resulting in very effective speed-ups for such design activities.

Scalable macromodeling of EM systems has attracted a lot of attention during recent years [1, 2, 3, 4, 5]. However, one of the key issues in these modeling approaches, rarely addressed in the literature, is the optimal selection of data samples in order to limit the total number of expensive EM simulations used as initial data for the model construction.

A recent work on sequential sampling of scattering parameter responses (S-responses) uses output $\mathbf{S}$-responses along with an exploration-exploitation-based approach [6]. In the exploration phase, which explores the design space for unidentified regions, a space filling technique is used to fill the design space uniformly, whereas in the exploitation phase, which identifies potentially interesting and dynamic regions of the design space, a local estimate of the gradient is used to select new samples.

This paper describes a novel sequential sampling algorithms for selecting the optimum number of samples such that accurate scalable models for parameterized system responses can be generated. The method presented here is quite different from the approach in [6], since the proposed algorithm is local and work on local hyper-rectangular regions of the design space making it applicable to several passivity-preserving scalable macromodeling methods $[2,3,4,5]$. Moreover, the algorithm can be implemented using tree structures, reducing the model evaluation time and making it portable to parallel computing platforms.

\section{Sequential sampling method}

A sequential sampling algorithm should be able to extract those system features which are most relevant in modeling its behavior. The design space which consists of parameters such as layout variables or substrate features is sampled so that the generated model is accurate over the complete design space. In this paper, a clear distinction is made between frequency and the rest of the design parameters as the former has special characteristics and is dealt separately. The selection of a suitable scalable macromodeling method to generate accurate models, the main features of the proposed sequential sampling algorithm and some other important aspects to be taken into consideration for an efficient sequential sampling are described in what follows.

Scalable macromodeling methods Different scalable macromodeling methods $[2,3,4,5]$ for which the proposed sequential sampling algorithm can be used, are briefly described here. These scalable macromodeling methods start from a set of data samples $\left\{(s, \vec{g})_{k}, \mathbf{H}(s, \vec{g})_{k}\right\}_{k=1}^{K_{\text {tot }}}$, which depend on the complex frequency $s=j \omega$ and several design variables $\vec{g}=\left(g^{(n)}\right)_{n=1}^{N}$. From these data samples, a set of frequency-dependent rational macromodels is built for some design space points by means of the Vector Fitting (VF) technique [7, 8, 9]. A pole-flipping scheme is used to enforce stability [7], while passivity assessment and enforcement can be accomplished using the standard techniques $[10,11]$. This initial step results in a set of stable and passive frequency dependent rational models, called root macromodels.

The set of root macromodels are then parameterized with respect to the design variables $\vec{g}$ with the help of stability and passivity preserving interpolations schemes [2, 3, 4, 5]. In [2], the root macromodels are interpolated at an input-output level which leads to the parameterization of residues with respect to $\vec{g}$. The modeling capability is further enhanced in the methods described in [3, 4] which interpolate system state-space matrices, there by parameterizing both poles and residues. However the methods in $[3,4]$ are sensitive to the issues related to the interpolation of state-space matrices. A recent passivitypreserving parametric macromodeling method has been proposed in [5], which uses an innovative passive interpolation of root macromodels at an input-output level by means of a set of amplitude and frequency scaling coefficients. It is able to parameterize both poles and residues indirectly, avoiding the interpolation of state-space matrices. In this paper, we use the scalable macromodeling method of [5] to guide the sequential sampling algorithm and to generate final scalable macromodels.

Core algorithm The proposed sequential sampling algorithm preserves the hyper-rectangular nature of the design space grid. From here on we refer to a hyper-rectangular region of the design space as a subspace. The algorithm assumes that, a scalable macromodel is prone to high modeling error at the center of the subspace such that the subspace is divided if certain requirements are not satisfied. For the description purpose, let us consider a case with two design parameters $\left(g^{(1)}, g^{(2)}\right)$, for 
which the design space is defined by four corners of a rectangle as shown in Fig. 1.a. A scalable macromodel is built using the corner frequency responses, and the accuracy of this initial macromodel is calculated by comparing the actual frequency response with the estimated frequency response at the center of the design space (shown by the light gray circle in Fig. 1.b). If the accuracy is not sufficient the design space is further divided as shown in Fig. 1.b. This generates four additional child subspaces or in general $2^{N}$ child subspaces, where, $N$ is the dimension of the design space. At this point the design space is further sampled at several locations as shown by the white circles in Fig. 1.b to preserve the hyper-rectangular nature of the design space. This is repeated until the modeling error satisfies a predefined error threshold $\Delta_{\text {err }}$.
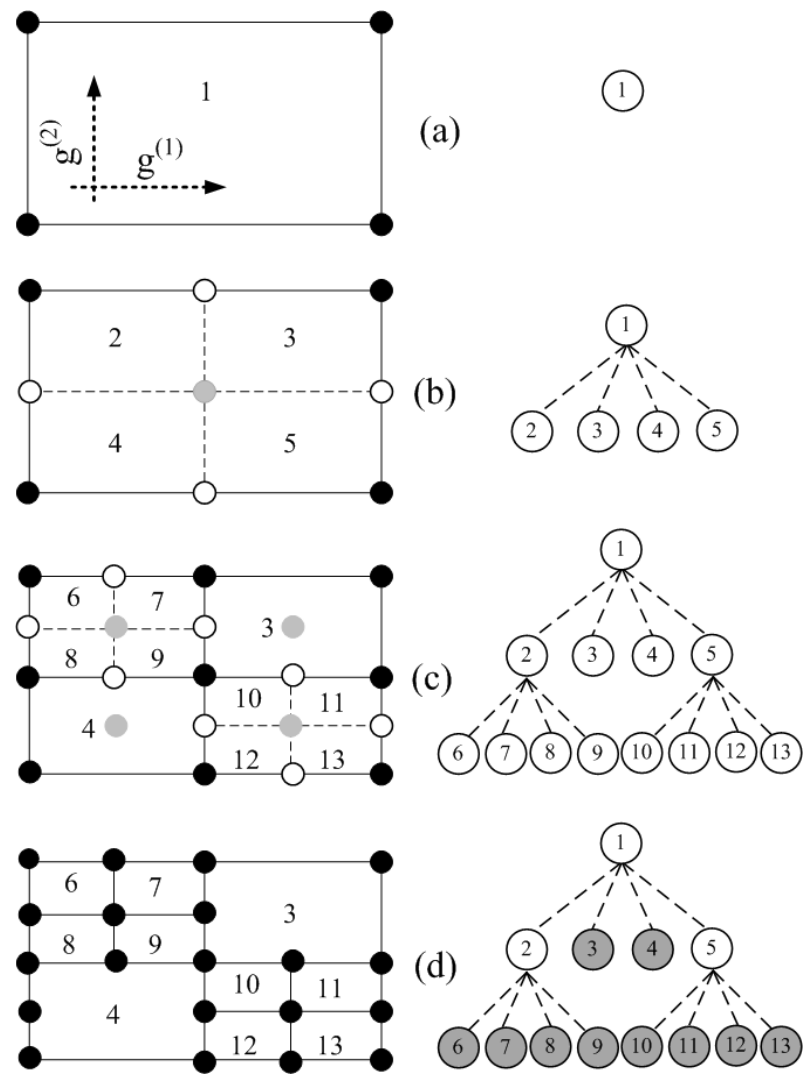

Figure 1: Evolution of the design space during sampling.

As shown in Fig.1, the algorithm can be represented in a tree structure with each circle representing a particular subspace. The algorithm begins by defining a single subspace as in Fig1.a and then generates four child subspaces numbered from 2 to 5 as in Fig. 1.b. An intermediate step is shown in Fig. 1.c, where the subspaces 2 and 5 divide to satisfy the error criteria, creating child regions 6 to 9 and 10 to 13 respectively. The tree structure of the final grid is shown in Fig. 1.d, where the gray circles represent the terminal subspaces of the design space. With a treebased implementation, there is a considerable gain in model evaluation time if macromodels based on local scalable macromodeling schemes $[2,3,4,5]$ are used, as the terminal subspace can be easily found through the branches. Another advantage is that the algorithm is portable to parallel computing platforms with further reduction of computing time.

Error and stopping criteria The number and distribution of the selected design space points depend on the error criteria selected, which play a critical role in the proposed sequential sampling. In this work, the mean absolute error (MAE)

$$
E_{r r}^{\mathrm{MAE}_{r}}(\vec{g})=\frac{\sum_{i=1}^{P_{i n}} \sum_{j=1}^{P_{\text {out }}} \sum_{k=1}^{N_{s}}\left|R_{i, j}\left(s_{k}, \vec{g}\right)-H_{i, j}\left(s_{k}, \vec{g}\right)\right|}{P_{\text {in }} P_{\text {out }} N_{s}}
$$

with number of input ports $P_{i n}$, output ports $P_{\text {out }}$ and frequency samples $N_{s}$ is used to compare original and modeled $\mathbf{S}$-responses at the center of each subspace.

Another important aspect of an efficient sequential sampling method is to use good stopping criteria to terminate the algorithm if the error thresholds are never satisfied. One obvious choice would be to limit the number of design space points generated by defining an upper bound. Another choice would be to use a lower bound on the resolution of the design space grid, e.g., by defining a lower threshold on the spatial increment along each design space direction $\delta \vec{g} \geq \delta \vec{g}_{\min }$. For example, in the case of layout parameters such as widths or lengths, it might be possible to know the manufacturing precision which defines $\delta \vec{g}_{\text {min }}$. When it is not known, a relative threshold $\delta \vec{g}_{\min }^{\text {rel }}$ can be defined such that:

$$
\delta \vec{g}^{\mathrm{rel}}=\frac{\text { Range of parameter in the subspace }}{\text { Range of parameter }} \geq \delta \vec{g}_{\text {min }}^{\text {rel }} .
$$

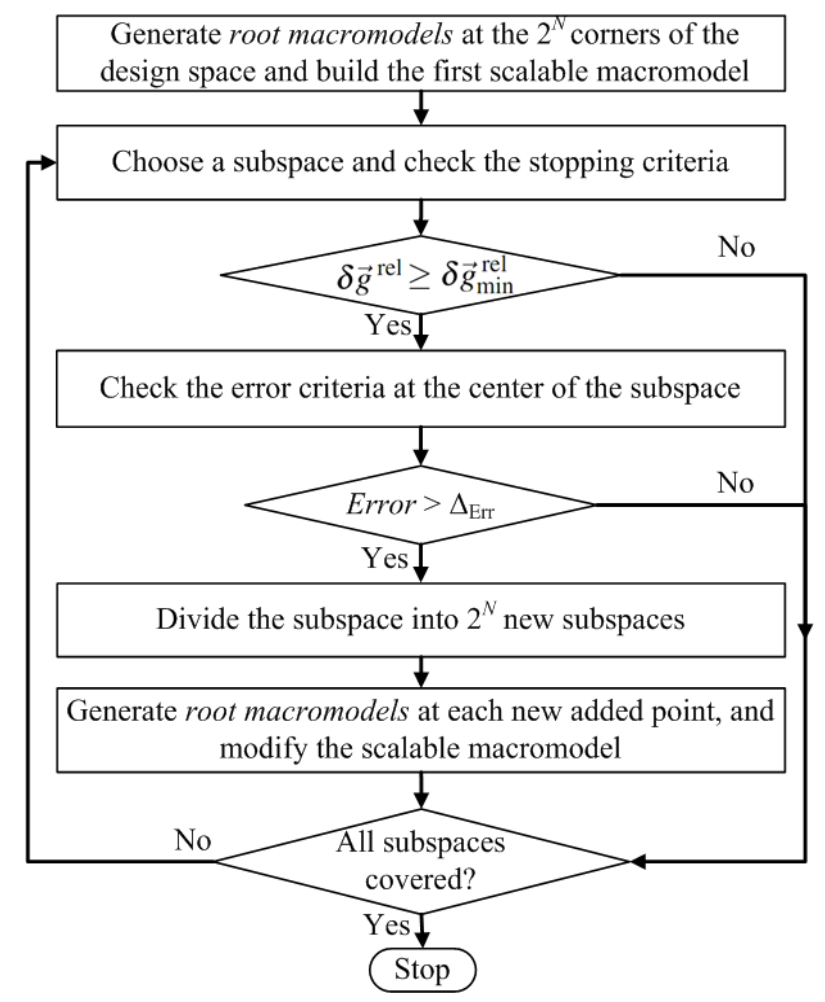

Figure 2: Flowchart of the proposed sequential sampling.

Fig. 2 shows the complete flowchart of the proposed sequential sampling algorithm. The algorithm first generates a 
set of root macromodels at the $2^{N}$ corner points which define the boundaries of the design space, which is used to build the first scalable macromodel. Then as shown in Fig. 1, the design space is refined using the sequential sampling algorithm. The algorithm terminates once all the subspaces are checked for the accuracy or the stopping criteria are met for those subspaces which violate the accuracy requirement.

\section{Numerical Simulation}

Four coupled microstrips, shown in Fig. 3, are modeled, where the spacing $S$ between the lines and the length $L$ of the lines are chosen as the design variables in addition to frequency. Table 1 shows the ranges of all parameters. The substrate is chosen with relative permittivity $\varepsilon_{r}=9.6$ and thickness equal to $0.125 \mathrm{~mm}$

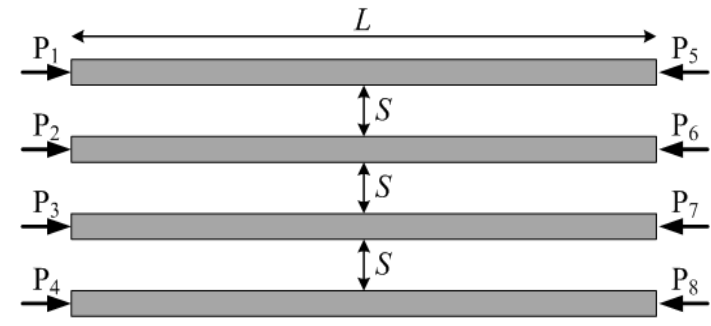

Figure 3: Top view of the layout of four coupled microstrips.

Table 1: Design variables of four coupled microstrips.

\begin{tabular}{|l|c|c|}
\hline Parameter & Min & Max \\
\hline Frequency $($ freq $)$ & $0 \mathrm{GHz}$ & $10 \mathrm{GHz}$ \\
Spacing $(S)$ & $0.05 \mathrm{~mm}$ & $0.15 \mathrm{~mm}$ \\
Length $(L)$ & $5 \mathrm{~mm}$ & $10 \mathrm{~mm}$ \\
\hline
\end{tabular}

The scattering matrix $\mathbf{S}(s, S, L)$ has been computed using the ADS Momentum $^{1}$ software, and the number of frequency samples has been chosen to be equal to $N_{s}=51$. Fig. 4 shows the parametric behavior of the magnitude of $\mathbf{S}_{11}(s, S, L)$ with respect to $L$ and frequency for $S=0.10 \mathrm{~mm}$. Similarly, the parametric behavior of the magnitude of $\mathbf{S}_{81}(s, S, L)$ with respect to $S$ and frequency is shown in Fig. 5 for $L=7.5 \mathrm{~mm}$.

Matlab R2010a ${ }^{2}$ is used to drive the ADS Momentum simulations to generate $\mathbf{S}$-responses which are then supplied to the proposed sequential sampling algorithm. For the stopping criterion, the minimum relative resolution $\delta \vec{g}_{\text {min }}^{\text {rel }}$ has been chosen to be equal to 0.05 .

Table 2 shows the number of design space points generated for varying levels of target accuracy in terms of the MAE (1). As discussed previously, in the algorithm the validation points are located at the geometrical center of each subspace (gray circles in Fig. 1).

The final design space generated using the proposed sequential sampling algorithm is shown in Fig. 6 for the accuracy level

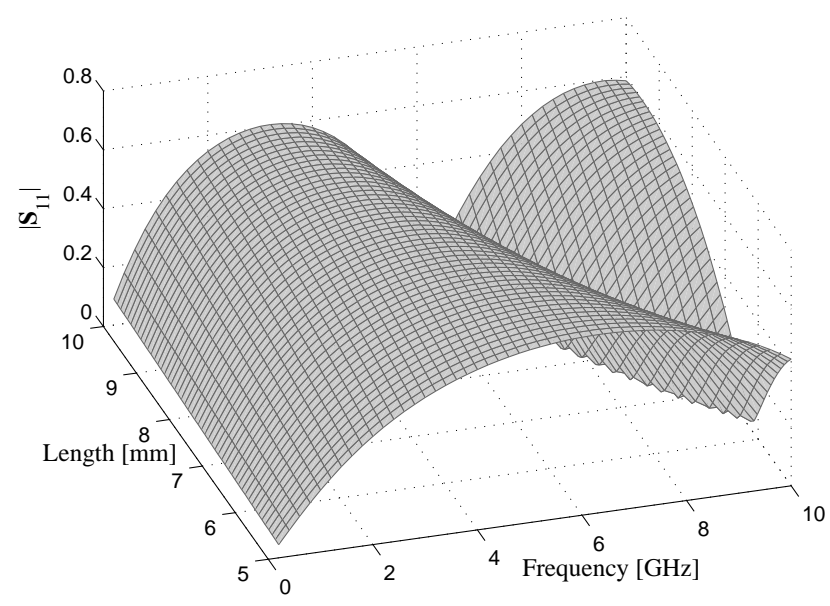

Figure 4: Parametric behavior of the magnitude of $\mathbf{S}_{11}$ for $S=$ $0.10 \mathrm{~mm}$.

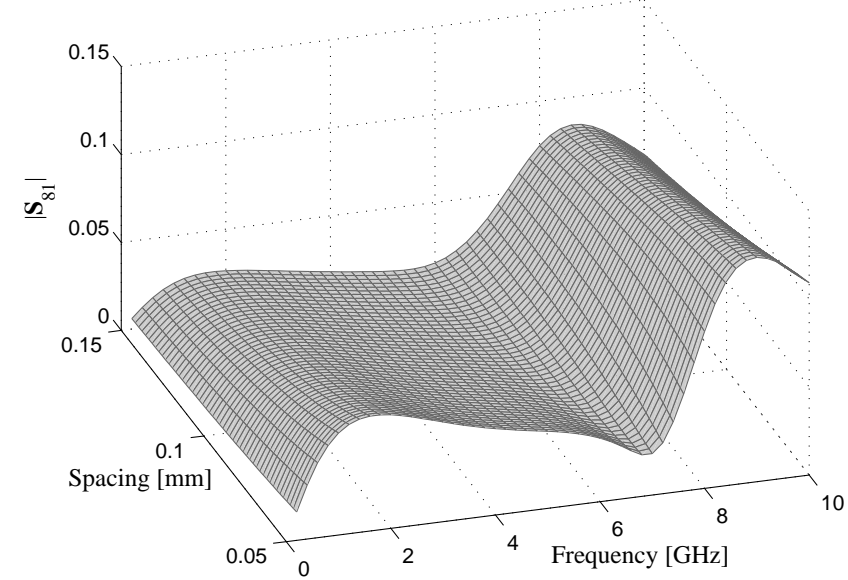

Figure 5: Parametric behavior of the magnitude of $\mathbf{S}_{81}$ for $L=$ $7.5 \mathrm{~mm}$.

Table 2: Sequential sampling results.

\begin{tabular}{|c|c|c|}
\hline \multicolumn{2}{|c|}{ Modeling accuracy } & \multirow{2}{*}{$\begin{array}{c}\text { Number } \\
\text { of points }\end{array}$} \\
\cline { 1 - 2 } Target $[\mathrm{dB}]$ & Achieved $[\mathrm{dB}]$ & 9 \\
-40 & -47.98 & 9 \\
-45 & -47.98 & 14 \\
-50 & -50.80 & 22 \\
-55 & -55.88 & 42 \\
-60 & -60.07 & \\
\hline
\end{tabular}

${ }^{1}$ Momentum EEsof EDA, Agilent Technologies, Santa Rosa, CA.

${ }^{2}$ The Mathworks, Inc., Natick, MA, USA 
equal to $-60 \mathrm{~dB}$. Some of the S-matrix entries at one of the validation point (gray asterisk in Fig. 6) are plotted for the scalable macromodel and compared with the ADS momentum data in Fig. 7, showing the achieved accuracy.

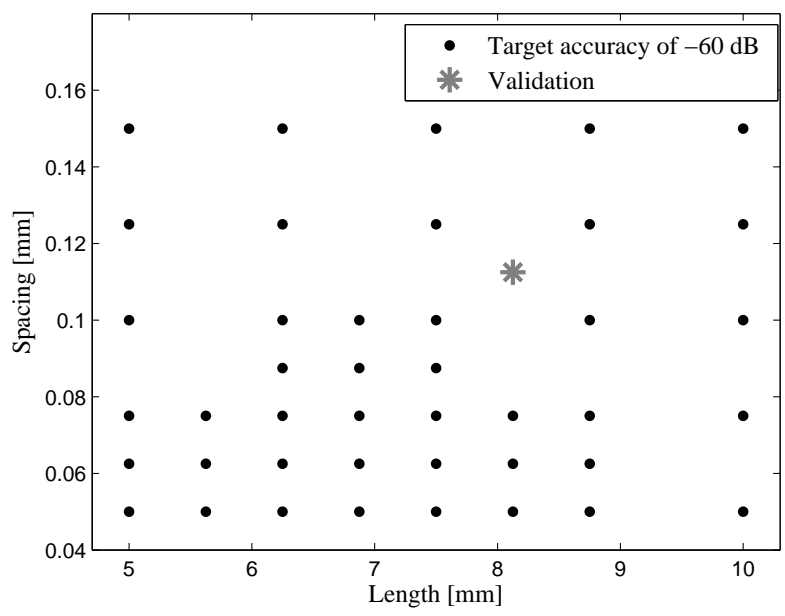

Figure 6: Design space generated by the proposed sequential sampling algorithm.

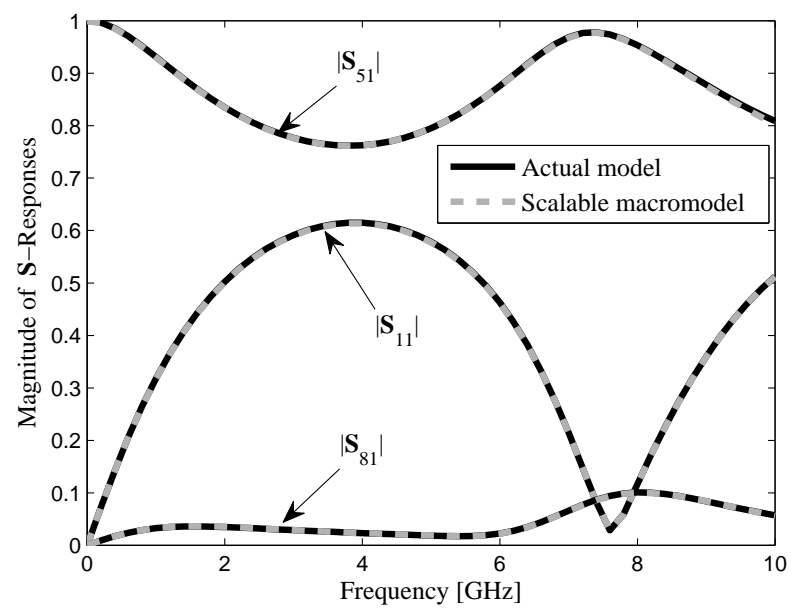

Figure 7: Magnitude of some S-matrix entries at $(S, L)=$ $(0.1125,8.125) \mathrm{mm}$.

\section{Conclusions}

We have presented a new sequential sampling algorithm for efficient and accurate scalable macromodeling of parameterized frequency responses of high-speed systems. The proposed sequential sampling algorithm was applied along with a scalable macromodeling method on a pertinent numerical example. The presented numerical results validate the proposed algorithm and shows its high modeling and sampling capabilities.

\section{Acknowledgments}

This work was supported by the Research Foundation Flanders (FWO).

\section{References}

[1] D. Deschrijver, T. Dhaene, and D. De Zutter, "Robust parametric macromodeling using multivariate orthonormal vector fitting," IEEE Transactions on Microwave Theory and Techniques, vol. 56, no. 7, pp. 1661 -1667, July 2008.

[2] F. Ferranti, L. Knockaert, and T. Dhaene, "Parameterized S-parameter based macromodeling with guaranteed passivity," IEEE Microwave and Wireless Component Letters, vol. 19, no. 10, pp. 608-610, Oct. 2009.

[3] P. Triverio, M. Nakhla, and S. Grivet-Talocia, "Passive parametric macromodeling from sampled frequency data," IEEE International Conference on Signal Propagation and Interconnects, pp. 117 -120, May 2010.

[4] F. Ferranti, L. Knockaert, T. Dhaene, and G. Antonini, "Passivity-preserving parametric macromodeling for highly dynamic tabulated data based on Lur'e equations," IEEE Transactions on Microwave Theory and Techniques, vol. 58, no. 12, pp. 3688 -3696, Dec. 2010.

[5] F. Ferranti, L. Knockaert, and T. Dhaene, "Passivitypreserving parametric macromodeling by means of scaled and shifted state-space systems," IEEE Transactions on Microwave Theory and Techniques, vol. 59, no. 10, pp. 2394 -2403, Oct. 2011.

[6] D. Deschrijver, K. Crombecq, H. Nguyen, and T. Dhaene, "Adaptive sampling algorithm for macromodeling of parameterized S-parameter responses," IEEE Transactions on Microwave Theory and Techniques, vol. 59, no. 1, pp. $39-45$, Jan. 2011.

[7] B. Gustavsen and A. Semlyen, "Rational approximation of frequency domain responses by vector fitting," IEEE Transactions on Power Delivery, vol. 14, no. 3, pp. 10521061, July 1999.

[8] B. Gustavsen, "Improving the pole relocating properties of vector fitting," IEEE Transactions on Power Delivery, vol. 21, no. 3, pp. 1587-1592, July 2006.

[9] D. Deschrijver, M. Mrozowski, T. Dhaene, and D. De Zutter, "Macromodeling of multiport systems using a fast implementation of the vector fitting method," IEEE Microwave and Wireless Components Letters, vol. 18, no. 6, pp. 1587-1592, June 2008.

[10] B. Gustavsen and A. Semlyen, "Fast passivity assessment for S-parameter rational models via a half-size test matrix," IEEE Transactions on Microwave Theory and Techniques, vol. 56, no. 12, pp. 2701-2708, Dec. 2008.

[11] B. Gustavsen, "Fast passivity enforcement for Sparameter models by perturbation of residue matrix eigenvalues," IEEE Transactions on Advanced Packaging, vol. 33, no. 1, pp. 257-265, Feb. 2010. 